\title{
Web-based Training for Implementing Evidence- Based Management of Postpartum Depression
}

\author{
Cynthia D. Baker, PhD, Hannah Kamke, BA, Michael W. O'Hara, PhD, and \\ Scott Stuart, $M D$
}

Background: Postpartum depression (PPD) is a significant mental health problem that is common in primary care and often under-recognized and under-treated.

Methods: We developed an educational website (www.step-ppd.com) to give primary care providers easy access to up-to-date information on PPD and evidence-based approaches for assessing and treating PPD. Three web-based modules include didactic information, case studies, interactive video clips, and links to additional resources.

Results: The Support and Training to Enhance Primary Care (STEP)-PPD website is a new and valuable resource for primary care providers that can be easily accessed online and provides accurate and current information on best practices for evidence-based management of PPD in primary care settings.

Conclusion: The STEP-PPD website is publicly available and free of charge. To register for and view the program, visit www.step-ppd.com. (J Am Board Fam Med 2009;22:588-589.)

Postpartum depression (PPD) is a serious mental health problem that is under-recognized and under-treated in the United States. ${ }^{1-3}$ Primary care settings are an ideal context in which to identify women at risk, and many providers are routinely screening for PPD. However, the effects of this screening are mitigated by limited training in the screening and treatment of PPD. For example, in one survey, $70 \%$ of family physicians reported routinely screening women at 6 weeks postpartum, but only $18 \%$ used a validated screening tool specifically designed for PPD. ${ }^{4}$ Moreover, to positively impact clinical outcomes, screening must be followed by evidence-based depression management,

This article was externally peer reviewed.

Submitted 30 December 2008; revised 29 April 2009; accepted 1 May 2009.

From Danya International, Inc., Silver Spring, MD (CDB, HK); the Department of Psychology (MWO) and the Department of Psychiatry (SS), University of Iowa, Iowa City.

Funding: This project has been funded in whole with Federal funds from the National Institute of Mental Health, National Institutes of Health, US Department of Health and Human Services, under contract number HHS-N-2782005-54995-C.

Conflict of interest: none declared.

Corresponding author: Cynthia D. Baker, PhD, Public and Behavioral Health Services, Danya International, Inc., 8737 Colesville Road, Suite 1100, Silver Spring, MD 20910 (E-mail: cbaker@danya.com). including accurate diagnosis and appropriate treatment and follow-up. ${ }^{5}$

To help fill this significant knowledge gap, in 2004 the National Institute of Mental Health solicited contract proposals through its Small Business Innovation Research program to develop for primary care providers training materials about the detection and treatment of PPD. This report describes a web-based training program developed under this solicitation, now publically available and free of charge.

\section{Description of the Web-Based Training Program}

Support and Training to Enhance Primary Care for Postpartum Depression (STEP-PPD) ${ }^{6}$ is a web-based training program designed to educate primary care providers about evidence-based screening, diagnosis, treatment, and referral for PPD. The primary goal is to increase providers' general knowledge about these elements of PPD. STEP-PPD also supports providers in implementation of evidence-based approaches for management of PPD in clinical practice. The target audience of STEP-PPD includes primary care providers across several fields. At registration, users are prompted to input information about their field (eg, family medicine, pediatrics, obstetrics/gynecol- 
ogy); profession (eg, physician, nurses, social workers, physician assistant); and many relevant special patient population characteristics (eg, race/ethnicity, residence in a rural region, adolescent mothers, women who used assisted reproductive technologies or gave birth to multiples). The content of the web-based training program is then customized for the user based on these variables.

The Web-based program contains the following 3 modules. (1) Understanding PPD presents information about the symptoms, etiology, and impact of PPD among women of different cultures, ethnicities, and geographic locations. The information reflects empirical research and clinical experience and serves as an introduction to PPD and other postpartum emotional difficulties. (2) Assessing PPD provides information and resources needed to implement evidence-based screening and assessment for PPD. Recommended approaches are designed to overcome common challenges faced by providers, including limited time for patient visits, competing time demands, and lack of adequate resources. (3) Treating PPD covers the guidelines for treatment of PPD in primary care settings using pharmacological and nonpharmacological interventions.

Each module provides detailed learning objectives, multiple case studies, interactive video clips, and links to additional resources. Case studies depict patient scenarios typically encountered in practice, and interactive video clips model provider interactions with postpartum women. In an effort to support implementation of evidence-based management practices, downloadable and printable resources such as patient education materials, screen- ing tools with parameters for scoring, as well as techniques for engaging patients, are included. Although the web-based training is publicly accessible and free of charge, Continuing Medical Education units and some supplementary products (eg, personal digital assistant and print versions of a Clinician's Pocket Guide) are available for a small fee.

To register for and view the program, visit www. step-ppd.com. ${ }^{6}$

The authors thank Adam Haim, PhD, of the National Institute of Mental Health, and Enid Light, PhD, before him, for support and guidance throughout this project.

\section{References}

1. O'Hara MW. Postpartum depression: causes and consequences. New York: Springer-Verlag; 1995.

2. Segre LS, O'Hara MW. The status of postpartum depression screening in the United States. In: Henshaw C, Elliott S, eds. Screening for perinatal depression. London: Jessica Kingsley; 2005:83-9.

3. Wisner KL, Logsdon MC, Shanahan BR. Webbased education for postpartum depression: conceptual development and impact. Arch Womens Ment Health 2008;11(5-6):377-85.

4. Seehusen DA, Baldwin LM, Runkle GP, Clark G. Are family physicians appropriately screening for postpartum depression? J Am Board Fam Pract 2005; 18:104-12.

5. Gjerdingen DK, Yawn, BP. Postpartum depression screening: importance, methods, barriers, and recommendations for practice. J Am Board Family Practice 2007;20:280-8.

6. Danya International, Inc. Support and training to enhance primary care for postpartum depression (STEP-PPD). Available at: www.step-ppd.com. Accessed July 4, 2009. 